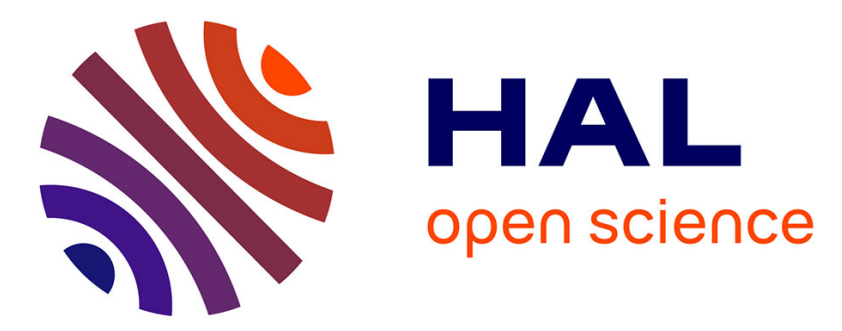

\title{
Role of the electron-phonon coupling on the thermal boundary conductance of metal/diamond interfaces with nanometric interlayers
}

Maïté Blank, Gionata Schneider, Jose Ordonez-Miranda, Ludger Weber

\section{- To cite this version:}

Maïté Blank, Gionata Schneider, Jose Ordonez-Miranda, Ludger Weber. Role of the electron-phonon coupling on the thermal boundary conductance of metal/diamond interfaces with nanometric interlayers. Journal of Applied Physics, 2019, 126 (16), pp.165302. 10.1063/1.5115823 . hal-02332626

\section{HAL Id: hal-02332626 \\ https://hal.science/hal-02332626}

Submitted on 24 Oct 2019

HAL is a multi-disciplinary open access archive for the deposit and dissemination of scientific research documents, whether they are published or not. The documents may come from teaching and research institutions in France or abroad, or from public or private research centers.
L'archive ouverte pluridisciplinaire HAL, est destinée au dépôt et à la diffusion de documents scientifiques de niveau recherche, publiés ou non, émanant des établissements d'enseignement et de recherche français ou étrangers, des laboratoires publics ou privés. 


\section{Role of the electron-phonon coupling on the thermal boundary conductance of metal/diamond interfaces with nanometric interlayers}

Cite as: J. Appl. Phys. 126, 165302 (2019); https://doi.org/10.1063/1.5115823

Submitted: 21 June 2019 . Accepted: 08 October 2019 . Published Online: 24 October 2019

Maïté Blank, Gionata Schneider, Jose Ordonez-Miranda (D), and Ludger Weber (D)

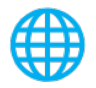

\section{Challenge us.} What are your needs for periodic signal detection?

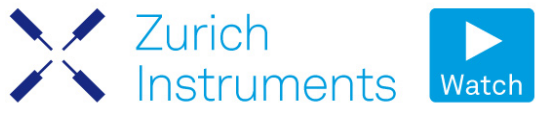

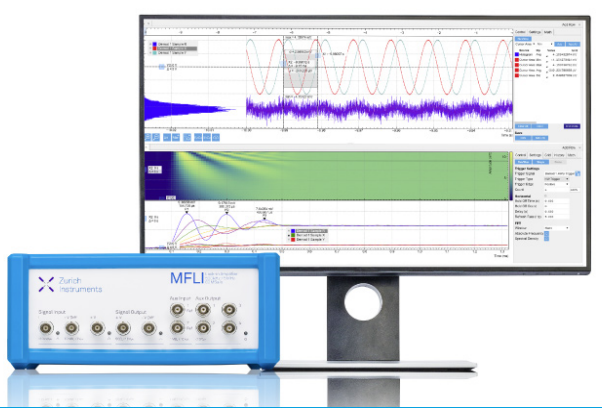

126, 165302 


\title{
Role of the electron-phonon coupling on the thermal boundary conductance of metal/diamond interfaces with nanometric interlayers
}

Cite as: J. Appl. Phys. 126, 165302 (2019); doi: 10.1063/1.5115823

Submitted: 21 June 2019 . Accepted: 8 October 2019.

Published Online: 24 October 2019

Maïté Blank, ${ }^{7, a)}$ Gionata Schneider, ${ }^{7}{\text { Jose Ordonez-Miranda, }{ }^{2} \text { (D) and Ludger Weber }}^{1, b)}$ (iD)

\begin{abstract}
AFFILIATIONS
'Laboratory of Mechanical Metallurgy, Ecole Polytechnique Fédérale de Lausanne, CH-1015 Lausanne, Switzerland

${ }^{2}$ Institut Pprime, CNRS, Université de Poitiers, ISAE-ENSMA, F-86962 Futuroscope Chasseneuil, France
\end{abstract}

${ }^{a)}$ Author to whom correspondence should be addressed: maite.blank@epfl.ch

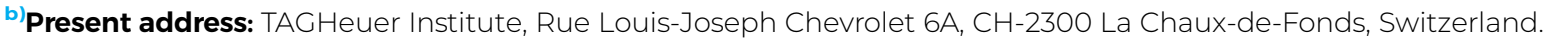

\begin{abstract}
Thermal boundary conductance (TBC) of the Ag/diamond and Au/diamond interfaces with a nanometer-thick interface layer of either nickel or molybdenum is measured by time domain thermoreflectance and modeled based on a 3-layer two-temperature model (31-TTM). The rationale for this study is to critically assess the role of the electron-phonon coupling factor of the interlayer along with its thickness on the TBC. It is shown that the TBC of both systems rapidly increases with the interlayer thickness until reaching a stable plateau for thicknesses greater than $1.5 \mathrm{~nm}$. The plateau average value is $15 \%-25 \%$ lower than the intrinsic TBC between the interlayer material and the diamond substrate. This behavior and values of the TBC of both systems are in good agreement with the predictions of the 31-TTM. The predictability of this model is also analyzed for a $\mathrm{Cu}$ interlayer inserted at $\mathrm{Au} / \mathrm{silicon}$ interfaces with thicknesses ranging from 1.5 to $20 \mathrm{~nm}$. While the room temperature TBC of this system is well described by the 3l-TTM, the values measured at $80 \mathrm{~K}$ can only be predicted by the 3l-TTM, provided that the interlayer electron-phonon coupling factor is reduced by a factor of 2, as was experimentally observed for Ag and $\mathrm{Au}$. The obtained experimental results along with the proposed model can thus be useful for tailoring the TBC of metallic interfaces in a wide range of temperatures.
\end{abstract}

Published under license by AIP Publishing. https://doi.org/10.1063/1.5115823

\section{INTRODUCTION}

With the continuous miniaturization of devices with enhanced rates of operation, the efficient management of heat transfer has become very challenging due to the overheating, low reliability, and reduced lifetimes of the involved nanomaterials. One of the key parameters driving the thermal performance of these devices is the finite thermal boundary conductance (TBC) $\left(h_{\mathrm{BD}}\right)$ between their different components, whose interfaces usually exhibit temperature jumps $\Delta T$ given by $\Delta T=J / h_{\mathrm{BD}}$, with $J$ being the heat flux across them. Typical values of the TBC for metal/dielectric interfaces are in the range of (10-800) $\mathrm{MW} / \mathrm{m}^{2} \mathrm{~K}^{1}$ and its importance can conveniently be evaluated through the ratio $h_{\mathrm{BD}} \cdot d / \kappa$ between the product $h_{\mathrm{BD}} \cdot d$ and the bulk thermal conductivity $(\kappa)$ of the interlayer material of thickness $d$. This ratio is similar to the Biot number in macroscopic heat transfer and indicates that the interface becomes progressively the main resistance to the heat flow for $h_{\mathrm{BD}} \cdot d / \kappa \leq 1$, which is, for example, often the case in laser diodes. ${ }^{2}$ This is the reason why extensive research efforts were carried out for enhancing the TBC, whose values are determined by the materials on either side of the interface ${ }^{1,3}$ and increase with the strength of the interfacial bonding ${ }^{4-6}$ and/or by reducing the interfacial roughness. ${ }^{7-9}$

In order to tailor the values of the TBC between a metallic film and its substrate, the influence of a thin interlayer with bridging properties inserted at their interface was studied in several works. By inserting $\mathrm{Ti}$ interlayers at both $\mathrm{Al} /$ graphite $^{10}$ and $\mathrm{Au} / \mathrm{Si}^{11}$ interfaces, the TBC was significantly enhanced, which was attributed to an improved interfacial bonding. Similarly, Wang et al. ${ }^{12}$ observed that $\mathrm{Al}$ and $\mathrm{Pt}$ interlayers with thicknesses ranging from 10 to $100 \mathrm{~nm}$ are able to enhance the TBC of $\mathrm{Au} / \mathrm{Si}$ interfaces, which was attributed to the electron-phonon coupling strengthening. Based on much 


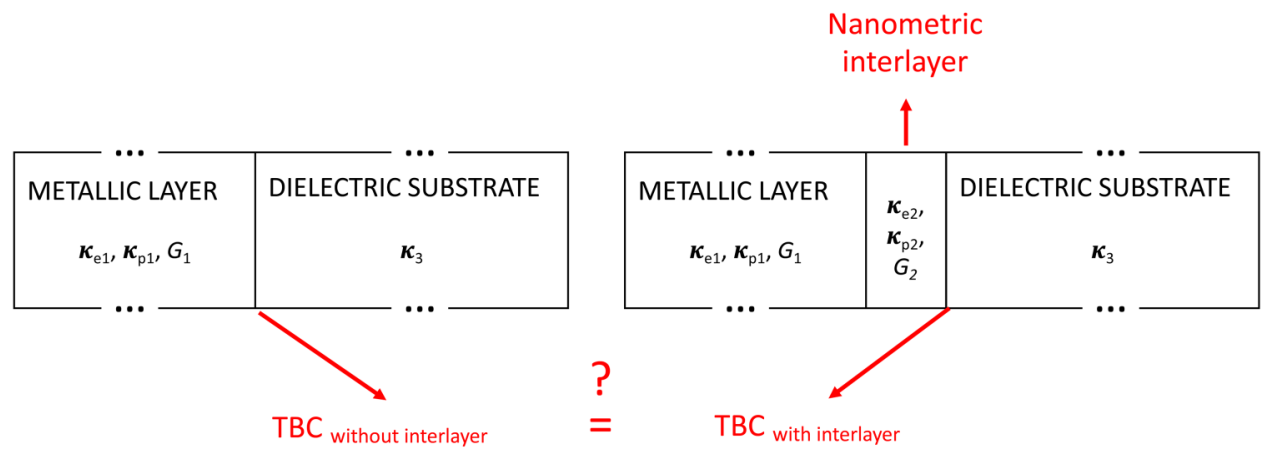

FIG. 1. Structure of the samples studied within this work. The influence of a thin interlayer, the thickness of which is varied from 1.5 to roughly $20 \mathrm{~nm}$, on the TBC at the interface between the metallic stack and the dielectric substrate is compared to the TBC obtained at the metal/dielectric interface when no interlayer is present. thinner $(0.3-8 \mathrm{~nm})$ interlayers of copper and chromium inserted at the $\mathrm{Au} / \mathrm{Al}_{2} \mathrm{O}_{3}$ interface, Jeong et al. ${ }^{13}$ showed that the TBC monotonically increases with the interlayer thickness, before reaching a plateau. Similar results were recently observed by our group ${ }^{14}$ for copper interlayers $(1.5-20 \mathrm{~nm})$ inserted at the $\mathrm{Au} / \mathrm{Si}, \mathrm{Au} / \mathrm{Al}_{2} \mathrm{O}_{3}$, and $\mathrm{Au} /$ diamond interfaces. The interpretation of the physical mechanisms generating this TBC behavior was, however, slightly different in both cases. By assuming that the interlayer contribution to the overall thermal transport across the interface is determined by phonons with wavelengths smaller than its thickness, Jeong et al. ${ }^{13}$ fitted a modified diffuse mismatch model (DMM) to their experimental results. By contrast, our results were described by means of a thermal resistance generated by the electron-phonon coupling inside the interlayer. ${ }^{14}$ This additional resistance decreases as the interlayer thickness increases such that the higher the electron-phonon coupling, the thinner the interlayer thickness required to reach the TBC plateau.

The purpose of this work is to rigorously assess this latter statement by measuring and modeling the TBC of metal/dielectric interfaces with ultrathin interlayers involving strong and weak electron-phonon coupling, as depicted in Fig. 1. This is done by comparing the experimental TBC values to the theoretical predictions of the simplified model presented in Ref. 14 and a refined one based on the two-temperature model applied to a three-layer system (31-TTM). This analytical 31-TTM explicitly takes into account the effect of the interlayer thickness on the TBC and its predictions are in good agreement with various experimental data even at $80 \mathrm{~K}$, provided that the interlayer electron-phonon coupling is reduced by a factor of two with respect to its value at room temperature.

\section{EXPERIMENTAL METHOD}

\section{A. Sample preparation and characterization}

Ag-Ni-diamond, Au-Ni-diamond, and Ag-Mo-diamond stacks were all fabricated by following the procedure developed for $\mathrm{Au}-\mathrm{Cu}$-diamond stacks and presented in our previous work. ${ }^{14}$ All diamond substrates consisted of high pressure high temperature synthetic stones (Ref: MWS L25 with [100] orientation, purchased from Element 6, Shannon, Co. Clare, Ireland), and their surface state was controlled through a succession of mechanical polishing steps ( $6 \mu \mathrm{m}, 3 \mu \mathrm{m}$, and $1 \mu \mathrm{m}$ diamond paste), followed by ultrasonic cleaning in acetone, ethanol, and isopropanol. Surface activation was achieved by using a Fischione 1020 oxygen plasma cleaner with a 3:1 Ar: $\mathrm{O}_{2}$ vol. gas composition. Each sample was exposed 15 min to the plasma and then immediately loaded into an Alliance-Concept DP 650 sputtering machine. Depending on the sample, a thin $\mathrm{Ni}$ or Mo layer was deposited on top of the diamond, before being covered by an 80 -nm-thick Ag or Au layer. A 20-nm-thick Cr layer was finally added on top of each sample to act as a transducer. A base pressure lower than $5 \times 10^{-6}$ mbar was used for all samples. In addition, $\mathrm{Au} /($ nanometer-thick $\mathrm{Cu}) / \mathrm{O}: \mathrm{C}$ samples presented in Ref. 14 were reused to measure the influence of ultrathin interlayers at liquid nitrogen temperature.

The thickness of each layer was measured by X-ray reflectivity (XRR), which is highly sensitive to the presence of ultrathin interlayers inserted between the main metallic layer and its substrate. ${ }^{14}$ A Panalytical diffractometer (model Empyrean, Malvern Panalytical Ltd.) equipped with a $\mathrm{Cu} \mathrm{K} \alpha \mathrm{X}$-ray source was used for all measurements, which were performed using the $\omega-2 \theta$ scanning mode with $\omega$ angles ranging from $0^{\circ}$ to $3^{\circ}$. The experimental curves were fitted by means of the GenX software, ${ }^{15}$ having as fitting parameters the layers' thickness, density, and roughness, as well as possible intermixing between two neighboring layers. The thickness of each interlayer was thus determined with an accuracy better than $\pm 0.3 \mathrm{~nm}$.

\section{B. Thermal boundary conductance measurement}

The TBC of each sample was measured by means of time domain thermoreflectance (TDTR), which consists of a pump-probe experiment. ${ }^{16,17}$ In a nutshell, a COHERENT Mira 900 operating at a wavelength of $785 \mathrm{~nm}$ with a repetition rate of $76 \mathrm{MHz}$ was used to generate the laser pulse train that was split into a pump and a probe part. Over its optical path, the pump was modulated at a frequency of $10.7 \mathrm{MHz}$ using an electro-optic modulator (EOM, Model 350-160, ConOptics Inc.), which is necessary for lock-in detection. It further went through a mechanical delay stage (M-531-PD1, Physik Instrument GmbH \& Co) that allowed sweeping delay times ranging from 0 to $4 \mathrm{~ns}$, which is necessary to record extensive cooling curves. Long (short) pass filters and half 
wave plates were inserted on both the pump and the probe optical path, respectively, to differentiate them in terms of wavelength and polarization. This so-called "two-tint" technique allows for significant noise reduction. At the end of their respective optical paths, both beams were focused on the same spot of the sample surface using a $10 \times$ microscope objective. There, the pump was heating the sample periodically, while the probe was monitoring the change in sample reflectivity, which is linearly dependent on the temperature, provided the temperature increase is not too large. This signal was sent back to a fast-photodiode (Thorlabs DET 10A), before being frequency-filtered, preamplified, and fed into a ZI-HF2 lock-in amplifier (Zurich Instruments, Zürich, Switzerland).

All measurements were carried out at a target beam power of $7 \mathrm{~mW}$ together with a target spot size of $4 \mu \mathrm{m}$ leading to a fluence per pulse of $0.2 \mathrm{~mJ} / \mathrm{cm}^{2}$. The exact value of beam power and spot size was recorded for each experiment using a power meter and a CMOS camera.

For low-temperature measurements, sample cooling down to $80 \mathrm{~K}$ was achieved using a Janis ST-500 cryostat mounted on a 5 -axes stage that was continuously cooled down with liquid nitrogen using a Janis Supertran transfer line, which were both vacuum insulated. The transfer line was pumped down to $1 \times 10^{-5} \mathrm{mbar}$ on a regular basis before being sealed. The cryostat was pumped down to values better than $1 \times 10^{-6}$ mbar, on a daily basis and sealed right before starting experiments to avoid vibrations that would prevent proper measurements. A LakeShore 336 temperature controller was used to achieve precise temperature control over the whole experiment duration.

The TBC values were extracted from the calculated $X / Y$ ratio, with $X$ and $Y$ corresponding to the in-phase and out-of-phase signal extracted from the lock-in amplifier, using the scheme proposed by Cahill. ${ }^{18}$ The input parameters were the thickness, thermal conductivity, and heat capacity of each layer. The metallic layers' thickness values were taken from XRR measurements. Their thermal conductivity and heat capacity, as well as the diamond heat capacity, were taken from the literature ${ }^{19-23}$ (see Table I). Finally,

TABLE I. Thermal conductivity and heat capacity values used to extract TBC values out of the X/Y TDTR signal.

\begin{tabular}{lccc}
\hline \hline Metal & $\begin{array}{c}\text { Temperature } \\
(\mathrm{K})\end{array}$ & $\begin{array}{c}\text { Thermal conductivity } \\
(\mathrm{W} / \mathrm{m} \mathrm{K})\end{array}$ & $\begin{array}{c}\text { Heat capacity } \\
\times 10^{6}\left(\mathrm{~J} / \mathrm{m}^{3} \mathrm{~K}\right)\end{array}$ \\
\hline $\mathrm{Ag}$ & 300 & $429^{\mathrm{a}}$ & $2.47^{\mathrm{a}}$ \\
$\mathrm{Au}$ & 300 & $317^{\mathrm{a}}$ & $2.49^{\mathrm{a}}$ \\
$\mathrm{Cr}$ & 300 & $93^{\mathrm{a}}$ & $3.21^{\mathrm{a}}$ \\
$\mathrm{Mo}$ & 300 & $138^{\mathrm{a}}$ & $2.56^{\mathrm{a}}$ \\
$\mathrm{Ni}$ & 300 & $91^{\mathrm{a}}$ & $3.95^{\mathrm{a}}$ \\
$\mathrm{Au}$ & 80 & $320^{\mathrm{b}}$ & $1.96^{\mathrm{c}}$ \\
$\mathrm{Cr}$ & 80 & $170^{\mathrm{b}}$ & $0.43^{\mathrm{d}}$ \\
$\mathrm{Cu}$ & 80 & $550^{\mathrm{b}}$ & $1.83^{\mathrm{e}}$ \\
\hline \hline
\end{tabular}

${ }^{\mathrm{a}}$ From Ref. 19.

${ }^{\mathrm{b}}$ From Ref. 20.

${ }^{c}$ From Ref. 22.

${ }^{\mathrm{d}}$ From Ref. 21.

${ }^{\mathrm{e}}$ From Ref. 23. the $\mathrm{TBC}$ and the diamond thermal conductivity were taken as fitting parameters. The diamond thermal conductivity was not set a priori, because it depends on the nitrogen content, which is recognized to vary from one stone to another, as well as within one stone, depending on whether the measurement was taken on a seed part or a secondary growth part. ${ }^{24}$ As shown in Fig. 2, a good signal to noise ratio, as well as satisfactory fit quality, was obtained in all cases.

A detailed analysis of the TDTR measurement sensitivity to the different parameters used is provided in the supplementary material.
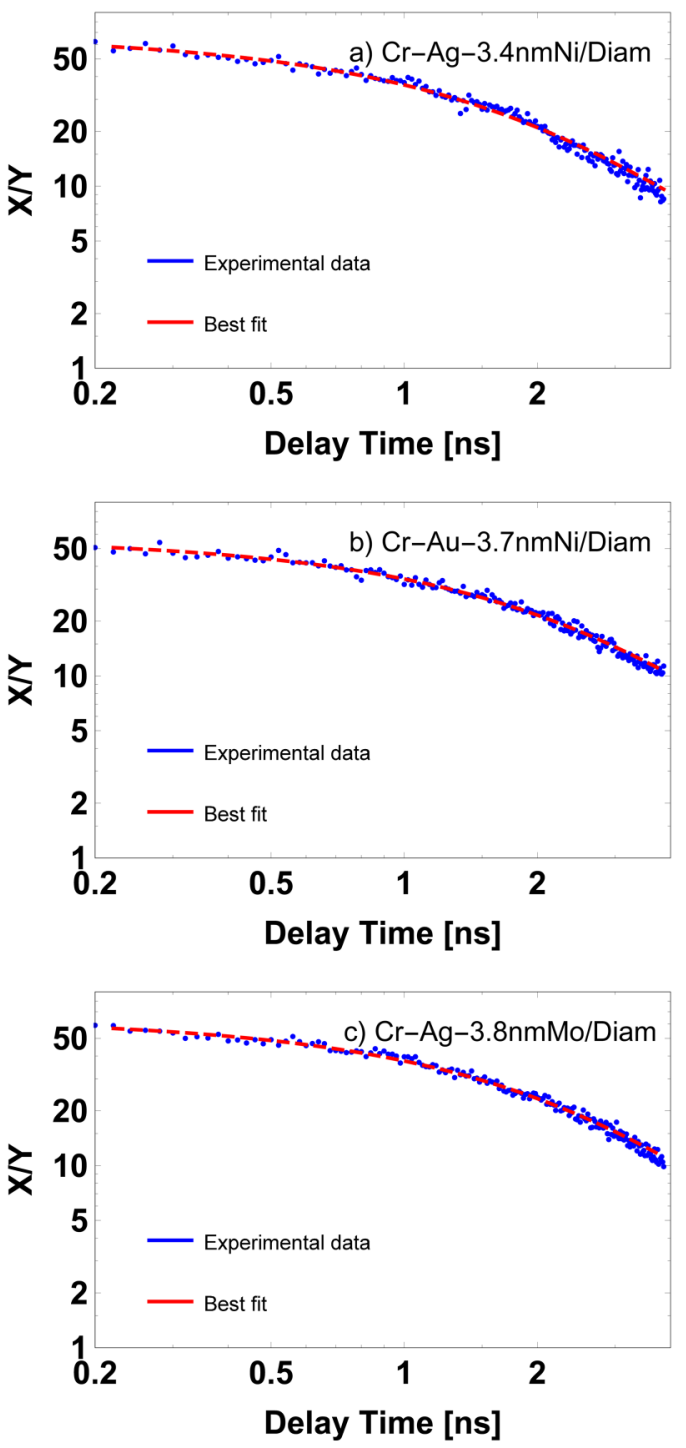

FIG. 2. TDTR raw data recorded for three samples of (a) Ag/(nanometer-thick $\mathrm{Ni}$ /diamond, (b) Au/(nanometer-thick Ni)/diamond, and (c) Ag/(nanometer-thick Mo)/diamond. The continuous lines stand for the best fits. 


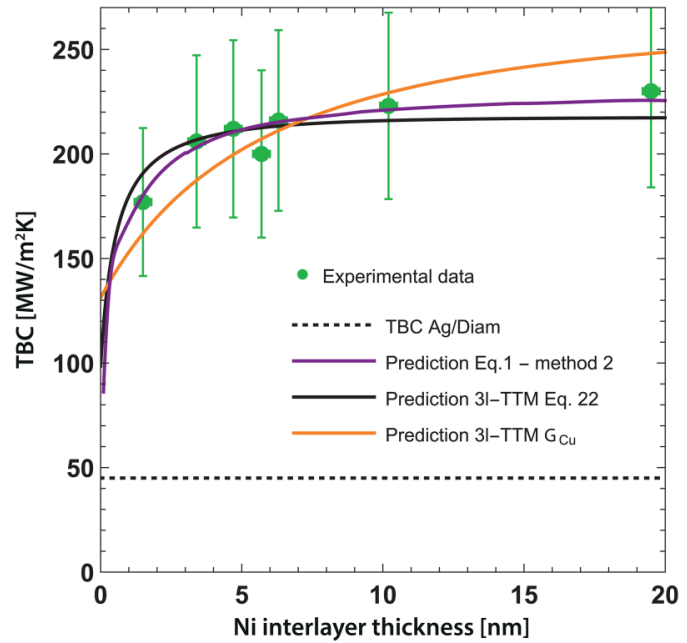

FIG. 3. Influence of a thin $\mathrm{Ni}$ interlayer on the $\mathrm{TBC}$ at the $\mathrm{Ag} / \mathrm{O}: \mathrm{C}$ interface together with the fits obtained with Eqs. (1) (purple) and (22) (black). The former fit was obtained using method 2, i.e., the best fit was calculated by allowing the Ag/diamond TBC value to vary within $20 \%$ of the value found in Ref. 25 , while the electron-phonon coupling factor was allowed to vary by a factor of two as compared to the value given in Table IV.

\section{RESULTS}

\section{A. TBC at Au/diamond and Ag/diamond interfaces with $\mathrm{Ni}$ and $\mathrm{Mo}$ interlayers}

The TBC values obtained for the $\mathrm{Ag} /($ nanometer-thick) $\mathrm{Ni}$ / diamond system are shown in Fig. 3. The TBC was observed to increase from $45 \mathrm{MW} / \mathrm{m}^{2} \mathrm{~K}^{25}$ to $177 \mathrm{MW} / \mathrm{m}^{2} \mathrm{~K}$ when inserting a $1.5 \mathrm{~nm} \mathrm{Ni}$ interlayer. For larger interlayers, a plateau was reached with an average value of $214 \mathrm{MW} / \mathrm{m}^{2} \mathrm{~K}$, i.e., $\sim 75 \%$ of the $\mathrm{Ni}$ / diamond $\mathrm{TBC}$ value of $290 \mathrm{MW} / \mathrm{m}^{2} \mathrm{~K}^{25}$

The TBC values measured for the $\mathrm{Au} /$ (nanometer-thick) $\mathrm{Ni}$ /diamond system are given in Fig. 4. The TBC was observed to increase from $76 \mathrm{MW} / \mathrm{m}^{2} \mathrm{~K}^{25}$ to $208 \mathrm{MW} / \mathrm{m}^{2} \mathrm{~K}$ when inserting a $1.5 \mathrm{~nm} \mathrm{Ni}$ interlayer. Unlike for the $\mathrm{Ag} /$ (nanometer-thick Ni)/diamond system, no further evolution is observed; the TBC has already reached its plateau value, which falls very close to the one obtained in the $\mathrm{Ag} /$ (nanometer-thick Ni)/diamond system (208 vs $214 \mathrm{MW} / \mathrm{m}^{2} \mathrm{~K}$ ).

Figure 5 shows the TBC values that were obtained for the $\mathrm{Ag} /$ (nanometer-thick) Mo/diamond system. The thinnest Mo interlayer that could be achieved was $3.8 \mathrm{~nm}$, which is large compared to the thinnest layers obtained for $\mathrm{Ni}$ or $\mathrm{Cu}$, which were rather close to $1.5 \mathrm{~nm}$. Consequently, no gradual increase in the TBC with the interlayer thickness could be observed. Instead, a roughly constant value of $189 \mathrm{MW} / \mathrm{m}^{2} \mathrm{~K}$ was reached for all samples tested, i.e., more than 4 times the TBC value measured at the $\mathrm{Ag} / \mathrm{diamond}$ interface and $85 \%$ of the Mo/diamond TBC value of $220 \mathrm{MW} / \mathrm{m}^{2} \mathrm{~K}^{25}$

\section{B. TBC at the Au/Si interface at $80 \mathrm{~K}$}

At $80 \mathrm{~K}$, the $\mathrm{TBC}$ at the $\mathrm{Au} / \mathrm{Si}$ interface was measured to be $47 \mathrm{MW} / \mathrm{m}^{2} \mathrm{~K}$, while it was measured to be $153 \mathrm{MW} / \mathrm{m}^{2} \mathrm{~K}$ at the $\mathrm{Cu} / \mathrm{Si}$ interface. Figure 6 shows the evolution of the TBC as a

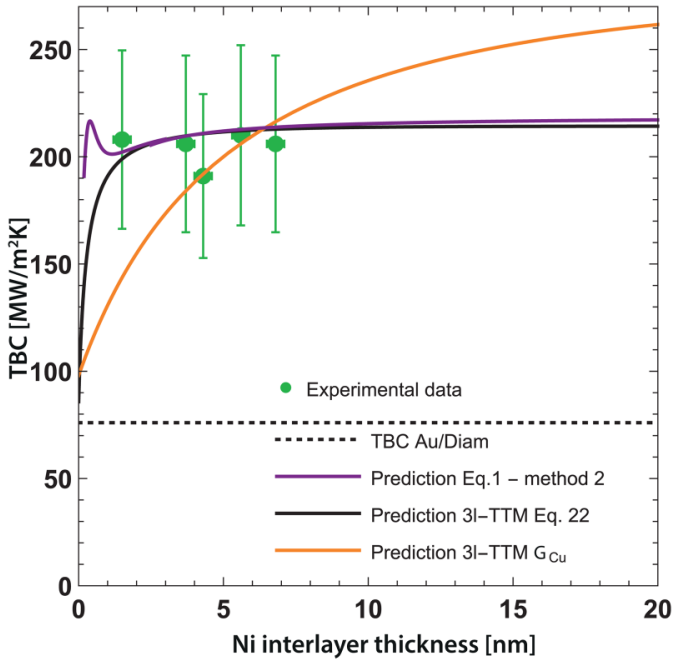

FIG. 4. Influence of a thin Ni interlayer on the TBC at the Au/O:C interface, same comments as for Fig. 2.

function of the copper interlayer thickness. A $1.5 \mathrm{~nm}$ interlayer is observed to already have a significant effect on the TBC by increasing it from $47 \mathrm{MW} / \mathrm{m}^{2} \mathrm{~K}(\mathrm{Au} / \mathrm{Si})$ to $80 \mathrm{MW} / \mathrm{m}^{2} \mathrm{~K}$. This value further increases for interlayer thicknesses up to roughly $10 \mathrm{~nm}$. A plateau is then reached with an average TBC value of $125 \mathrm{MW} / \mathrm{m}^{2} \mathrm{~K}$, which corresponds to $80 \%$ of the value achieved at the $\mathrm{Cu} / \mathrm{Si}$ interface.

\section{DISCUSSION}

\section{A. Deviations from Fourier's law in TDTR experiments}

A number of studies showed that TDTR measurements are likely to deviate from Fourier's law, especially for high-thermal

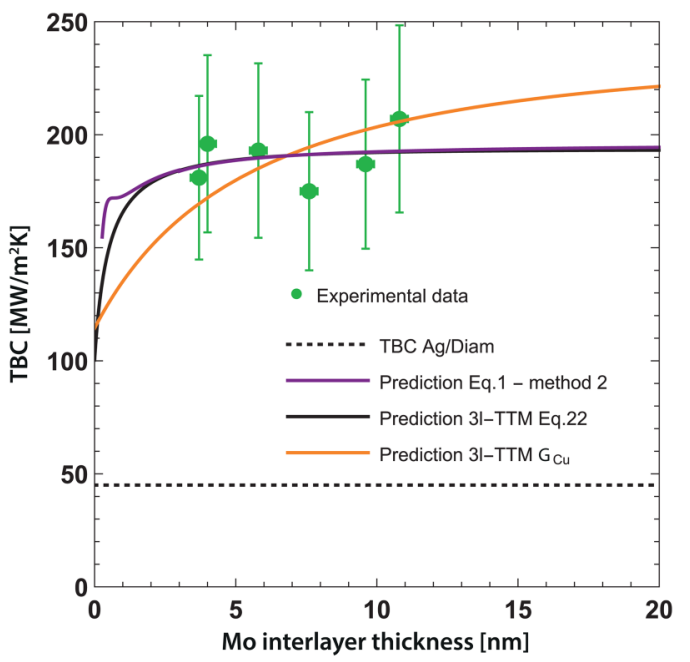

FIG. 5. Influence of a thin Mo interlayer on the TBC at the $\mathrm{Ag} / \mathrm{O}: \mathrm{C}$ interface, same comments as for Fig. 2. 


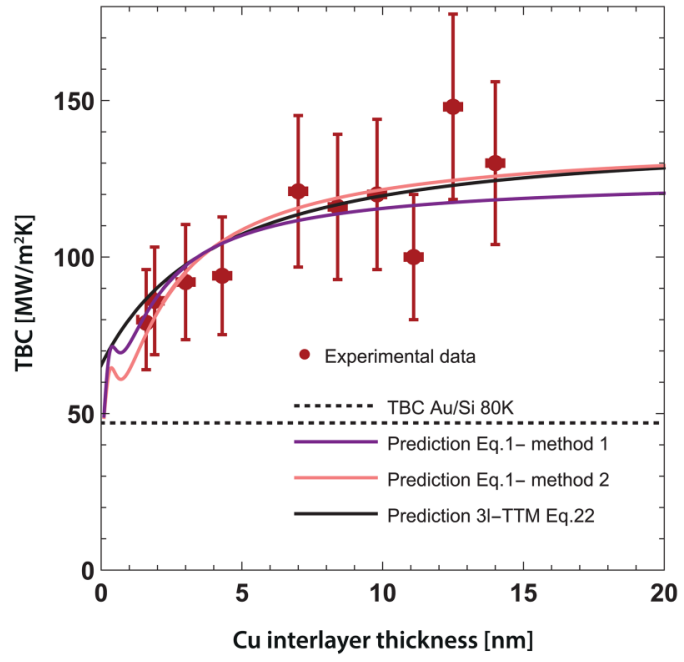

FIG. 6. Influence of a thin $\mathrm{Cu}$ interlayer on the TBC at the Au/Si interface measured at $80 \mathrm{~K}$ together with the fits obtained with Eqs. (1) (dotted purple and purple) and (22) (black). The former fit was obtained using method 2, i.e., the best fit was calculated by allowing the Au/diamond TBC value to vary within $20 \%$, while the electron-phonon coupling factor was allowed to vary by a factor of two as compared to the value given in Table IV.

conductivity crystals, such as diamond, for example. ${ }^{26-28}$ In a nutshell, these deviations are attributed to ballistic phonon transport in the sample and to a mismatch in the distribution of phonons that carry heat across the metal-dielectric interface. The existence of such deviations results in TDTR measurements to depend on the laser spot size and on the pump modulation frequency, which allows changing the characteristic length scale of the temperature gradient and thus modifies the relative amount of low-frequency phonons with a long mean free path that are not in local equilibrium with high-frequency phonons. This causes the apparent substrate thermal conductivity and interface thermal conductance derived from the thermal model to diminish when diminishing the characteristic length scale. The effect of Fourier's law failure, however, appears to have a much stronger impact on the thermal conductivity than on the thermal boundary conductance. ${ }^{26}$ In this last case, the error typically falls within the uncertainty of TBC values extracted from TDTR, which makes us believe that the procedures we used to extract out TBC values are sound. Furthermore, even if Fourier's law failure had a significant impact on the TBC values, we would expect all values within one system to be shifted by the same amount. The TBC dependence on the interlayer thickness is described in Sec. III, and the mechanisms controlling the TBC when inserting an interlayer at the interface between a metal and a dielectric would thus remain the same.

\section{B. Influence of the interlayer electron-phonon coupling factor on the TBC at metal/dielectric interfaces}

Earlier works performed on the influence of a thin interlayer on the TBC at metal/dielectric interfaces used interlayers made of

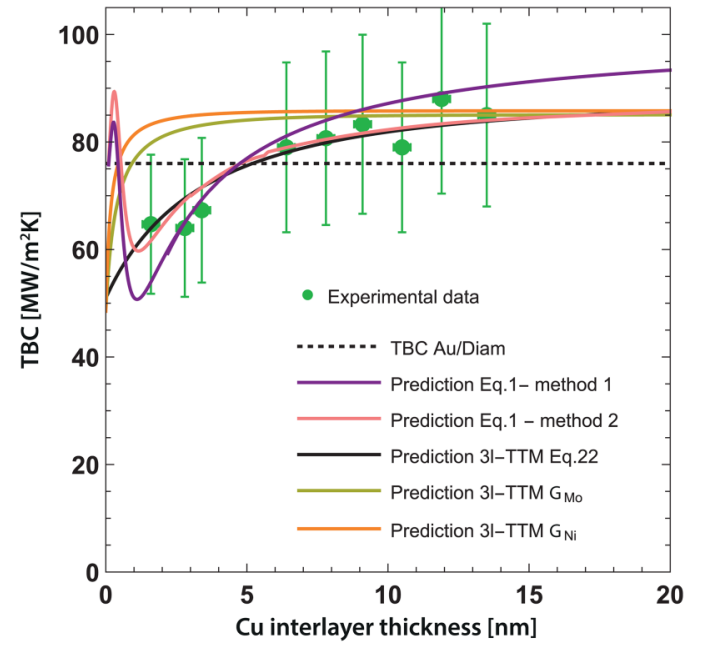

FIG. 7. Influence of a thin $\mathrm{Cu}$ interlayer on the TBC at the $\mathrm{Au} / \mathrm{O}: \mathrm{C}$ interface together with the fits obtained with Eqs. (1) (dotted purple and purple) and )22) (black). The dotted purple fit (method 1) was obtained assuming the TBC to be fixed, while the $G$ was fitted without any particular restriction. The solid purple fit was obtained using method 2, i.e., the best fit was calculated by allowing the Au/diamond TBC value to vary within $20 \%$ of the value found in Ref. 14 , while the electron-phonon coupling factor was set to half of the value given in Table IV. Adapted from Ref. 14.

copper. ${ }^{13,14}$ The TBC was observed to increase monotonically for interlayers up to roughly $10 \mathrm{~nm}$ before reaching a plateau at a value that is close to the interlayer/dielectric TBC value (see Figs. 7-9). Blank and Weber ${ }^{14}$ postulated that the TBC dependence on the interlayer thickness can be rationalized, assuming that the thermal

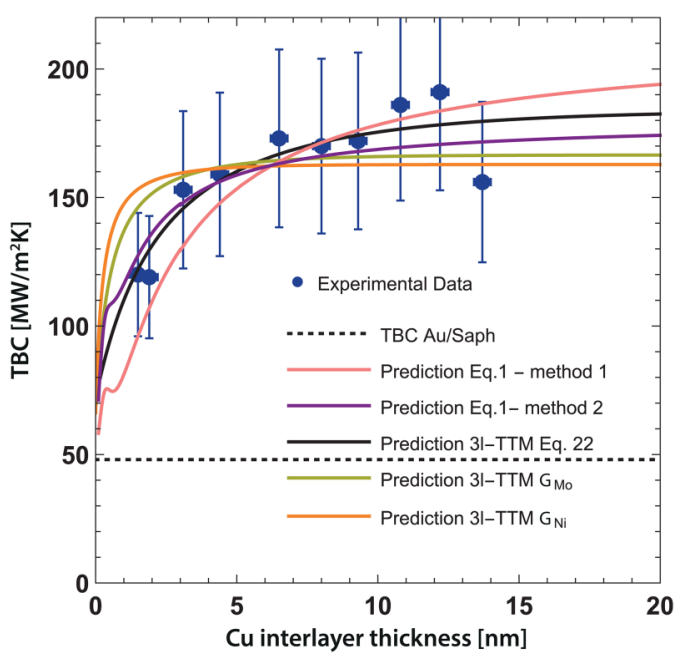

FIG. 8. Influence of a thin $\mathrm{Cu}$ interlayer on the $\mathrm{TBC}$ at the $\mathrm{Au} / \mathrm{Al}_{2} \mathrm{O}_{3}$ interface, same comments as for Fig. 6. Adapted from Ref. 14. 


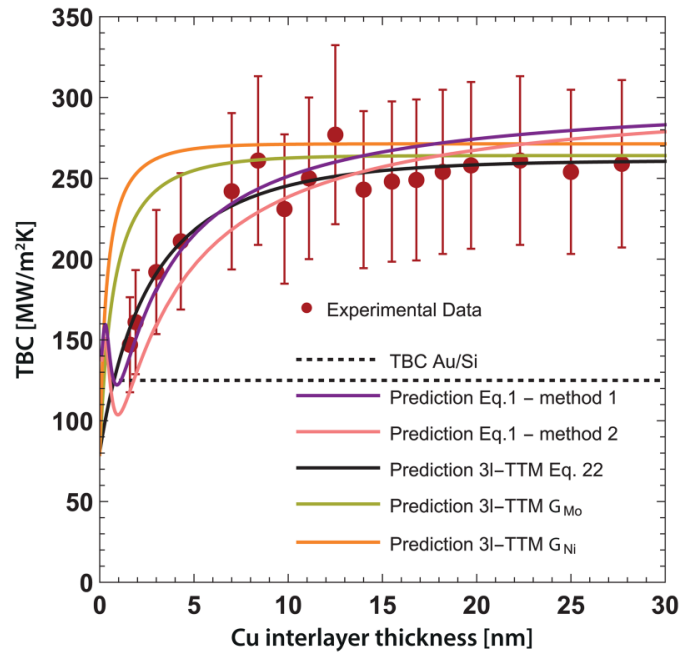

FIG. 9. Influence of a thin $\mathrm{Cu}$ interlayer on the TBC at the $\mathrm{Au} / \mathrm{Si}$ interface, same comments as for Fig. 6. Adapted from Ref. 14.

transport is controlled by three resistances in series, i.e., the metalmetal interfacial resistance $1 / h_{\mathrm{ee}}$, the metal-dielectric resistance $1 / h_{\mathrm{pp}}$, and a resistance within the interlayer that depends on its electron-phonon coupling factor $G$ and that becomes smaller as the interlayer gets thicker. A resistance in parallel was further added to account for a possible direct coupling between the phonons from the main metallic layer and the substrate. This last contribution is referred to as $h_{\text {tunneling }}{ }^{13,14}$ for lack of a better descriptor albeit the mechanism is not a quantum mechanical tunneling effect. The system total conductance is consequently expressed by

$$
h_{B D}=\left(\frac{1}{h_{\mathrm{ee}}}+\frac{1}{h_{\mathrm{pp}}}+\frac{1}{G \cdot l}\right)^{-1}+h_{\text {tunneling }},
$$

with $l$ being the interlayer thickness.

We first note that the contribution by phonon tunneling will decrease rapidly with the interlayer thickness and will be negligible for thicknesses larger than roughly $2 \mathrm{~nm}$. For the leading term in parenthesis of Eq. (1), a value independent of the layer thickness, $l$, i.e., a plateau in $h_{\mathrm{BD}}$, will be reached when the product of $G \cdot l$ gets very large compared to, both, $h_{\mathrm{ee}}$ and $h_{\mathrm{pp}}$. The onset of this plateau is hence expected to start at a very small interlayer thickness for a large interlayer electron-phonon coupling factor and to progressively move toward larger interlayer thickness with decreasing electron-phonon coupling factor. This statement can be critically assessed by comparing the results obtained for interlayers made of copper, which has a relatively weak electron-phonon coupling factor $\left[G_{\mathrm{Cu}}=(5-10) \times 10^{16} \mathrm{~W} / \mathrm{m}^{3} \mathrm{~K}^{29,30}\right]$, to the results obtained for interlayers made of nickel or molybdenum, which have a much stronger electron-phonon coupling constant $\left(G_{\mathrm{Ni}} \approx 100 \times 10^{16} \mathrm{~W} / \mathrm{m}^{3} \mathrm{~K}\right.$, $G_{\mathrm{Mo}} \approx 50 \times 10^{16} \mathrm{~W} / \mathrm{m}^{3} \mathrm{~K}^{29}$ ).

The TBC measured for $1.5 \mathrm{~nm}$-thick Ni interlayers inserted at the interface between $\mathrm{Ag}$ and diamond was shown to be slightly lower than the value measured for $3.4 \mathrm{~nm}$-thick interlayers, although the difference is comparable to the measurement uncertainty. This might indicate the presence of a gradual increase of the TBC over a very limited range of interlayer thicknesses. For interlayers larger than $1.5 \mathrm{~nm}$, a plateau is reached. For $\mathrm{Au} /($ nanometer-thick $\mathrm{Ni}$ )/diamond and $\mathrm{Ag} /($ nanometer-thick Mo)/ diamond samples, such a gradual increase of the TBC could not be observed. The plateau was reached even for the thinnest layer tested. This indicates that the increase of the TBC due to the $\mathrm{Ni}$ and Mo interlayers is much more abrupt than the TBC increase due to copper interlayers. This result is in qualitative agreement with the prediction given by Eq. (1).

A quantitative characterization of each system was achieved by fitting Eq. (1) to the experimental data. The conductance due to phonons "tunneling" describes the contribution of phonons coming from the gold layer with wavelengths longer than the interlayer thickness, which allows them to transmit their energy directly to the phonons in the substrate. This conductance is typically expected to reach its maximum for interlayer thicknesses close to zero before decreasing rapidly and eventually vanishes for interlayers thicker than roughly $2 \mathrm{~nm}$. Evaluating this contribution through the Debye model requires knowledge about the phonon maximum frequency, speed in the metal layer, and TBC at the metal/dielectric interface. These values are provided in Table II, while the details about calculations can be found in Ref. 14 .

The metal-metal interfacial conductance $h_{e e}$ was estimated by using the electronic version of the DMM developed by Gundrum et $a .^{33}$ and expressed by

$$
h_{\mathrm{ee}}=\frac{\gamma_{\mathrm{s}, 1} v_{\mathrm{f}, 1} \gamma_{\mathrm{s}, 2} v_{\mathrm{f}, 2}}{4\left(\gamma_{\mathrm{s}, 1} v_{\mathrm{f}, 1}+\gamma_{\mathrm{s}, 2} v_{\mathrm{f}, 2}\right)} T
$$

with $\gamma_{\mathrm{s}}$ being the Sommerfeld constant, $v_{\mathrm{f}}$ being the Fermi velocity of the materials on either side of the interface, and $T$ being the temperature. This model is heuristic and is built in analogy to the Diffuse Mismatch Model for phonons. It obeys the zero net charge condition but is not the only one to do so. Given the weak influence of the actual value of $h_{\mathrm{ee}}$ on the TBC determination of the interlayer-dielectric interface, we consider it as good as any other possible assumption leading to a $h_{\mathrm{ee}}$ value significantly larger than the TBC at the interlayer-dielectric interface. Table III summarizes the results obtained for the interfaces studied.

$h_{\mathrm{pp}}$ and $G$ were then extracted by evaluating the contribution of the three conductances in series $\left(h_{\mathrm{ee}}, h_{\mathrm{pp}}\right.$, and $\left.G \cdot l\right)$ from the experimental data using a best fit procedure. Therefore, $h_{\mathrm{pp}}$ values

TABLE II. Parameters used to evaluate the conductance due to phonon tunneling from the gold layer with $\omega_{\max }$ being the phonons maximum frequency, $v_{a v}$ being the average sound velocity, and $G_{e-p h}$, metal/diamond being the experimental TBC measured at the metal/diamond interface.

\begin{tabular}{lccc}
\hline \hline Metal & $\begin{array}{c}\omega_{\max } \\
(\mathrm{THz})^{31}\end{array}$ & $\begin{array}{c}v_{\mathrm{av}} \\
(\mathrm{m} / \mathrm{s})^{32}\end{array}$ & $\begin{array}{c}h_{p p \text {,metal-diamond }} \\
\left(\mathrm{MW} / \mathrm{m}^{2} \mathrm{~K}\right)^{25}\end{array}$ \\
\hline $\mathrm{Au}$ & 4.7 & 2128 & 45 \\
$\mathrm{Ag}$ & 5.1 & 2514 & 75 \\
\hline \hline
\end{tabular}


TABLE III. $1 / R_{\mathrm{e} 12}$ values calculated from the electronic equivalent of the DMM for the different metal-metal couples tested.

\begin{tabular}{|c|c|c|c|c|c|}
\hline Metal couple & $\begin{array}{c}\gamma_{\mathrm{s}, 1} \\
\left(\mathrm{~J} / \mathrm{m}^{3} \mathrm{~K}\right)^{34}\end{array}$ & $\times 10^{6}{ }^{v_{\mathrm{f}, 1}}(\mathrm{~m} / \mathrm{s})^{34}$ & $\begin{array}{c}\gamma_{\mathrm{s}, 2} \\
\left(\mathrm{~J} / \mathrm{m}^{3} \mathrm{~K}\right)^{34}\end{array}$ & $\times 10^{6}{ }^{v_{\mathrm{f}, 2}}(\mathrm{~m} / \mathrm{s})^{35}$ & $\begin{array}{l}h_{\mathrm{ee}}=1 / R_{\mathrm{e} 12} \\
\left(\mathrm{GW} / \mathrm{m}^{2} \mathrm{~K}\right)\end{array}$ \\
\hline $\mathrm{Ag}-\mathrm{Ni}$ & 62.8 & 1.39 & 1064 & 0.2 & 4.6 \\
\hline $\mathrm{Au}-\mathrm{Ni}$ & 71.5 & 1.4 & 1064 & 0.2 & 5.1 \\
\hline Ag-Mo & 62.8 & 1.39 & 214 & 0.9 & 4.5 \\
\hline
\end{tabular}

were taken from Ref. 25 and allowed to vary within $20 \%$, while $G$ was taken from Ref. 29 (see Table III) and allowed to vary by a factor of 2. A variability of $20 \%$ for $h_{\mathrm{pp}}$ value was allowed because it corresponds to the typical uncertainty attributed to TDTR measurements. As for the $G$ value, the factor of 2 reflects the variability of the values that are typically found in the literature. ${ }^{29,36-38}$ This procedure (later referred to as method 2) is slightly different from the one used in Ref. 14 (later referred to as method 1), in which $h_{\mathrm{pp}}$ was fixed and the $G$ was fitted without any particular restriction. This modification was necessary to allow for experimental data fitting using the 3l-TTM (see Sec. IV C) and thus to allow for a direct comparison between both methods. The results shown in Figs. 7-9 were fitted using both methods. Very similar predictions were obtained in both cases. As shown in Figs. 3-5, a good fit quality was obtained for all tested systems, which tends to confirm the importance of the interlayer electron-phonon coupling factor in the overall thermal transport at metal/(nanometer-thick) interlayer/dielectric interfaces. The initial TBC increase predicted by the model for very thin interlayers is an artifact that is attributed to the rough Debye approximation used to evaluate the contribution due to phonon "tunneling," i.e., the mechanism invoked by Jeong et al. ${ }^{13}$ in which phonons with a wavelength longer than the interlayer thickness are not affected by the interlayer. This was, however, found not to have a significant impact on our modeling results, which describe all the important features observed in the experiments, suggesting that the model captures the main mechanisms involved in the TBC evolution when a thin interlayer is added between a metal and a dielectric.

\section{Limitations of the previously presented model}

The underlying picture of heat transfer leading to Eq. (1) is schematically represented in Fig. 10(a). The overall drop in temperature across the interface consists of a drop $\Delta T_{\text {ee }}$, a drop $\Delta T_{\text {ep }}$ in the

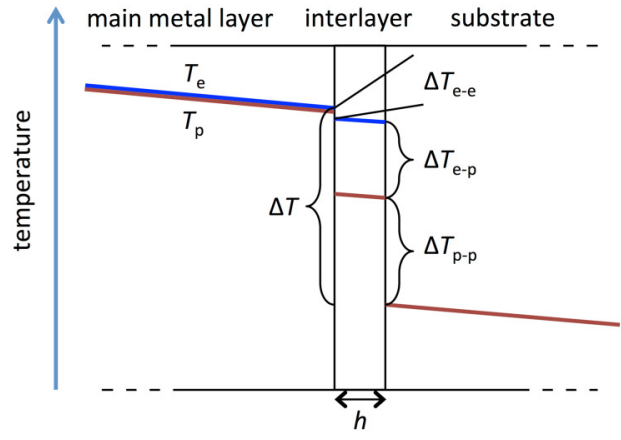

a) b)
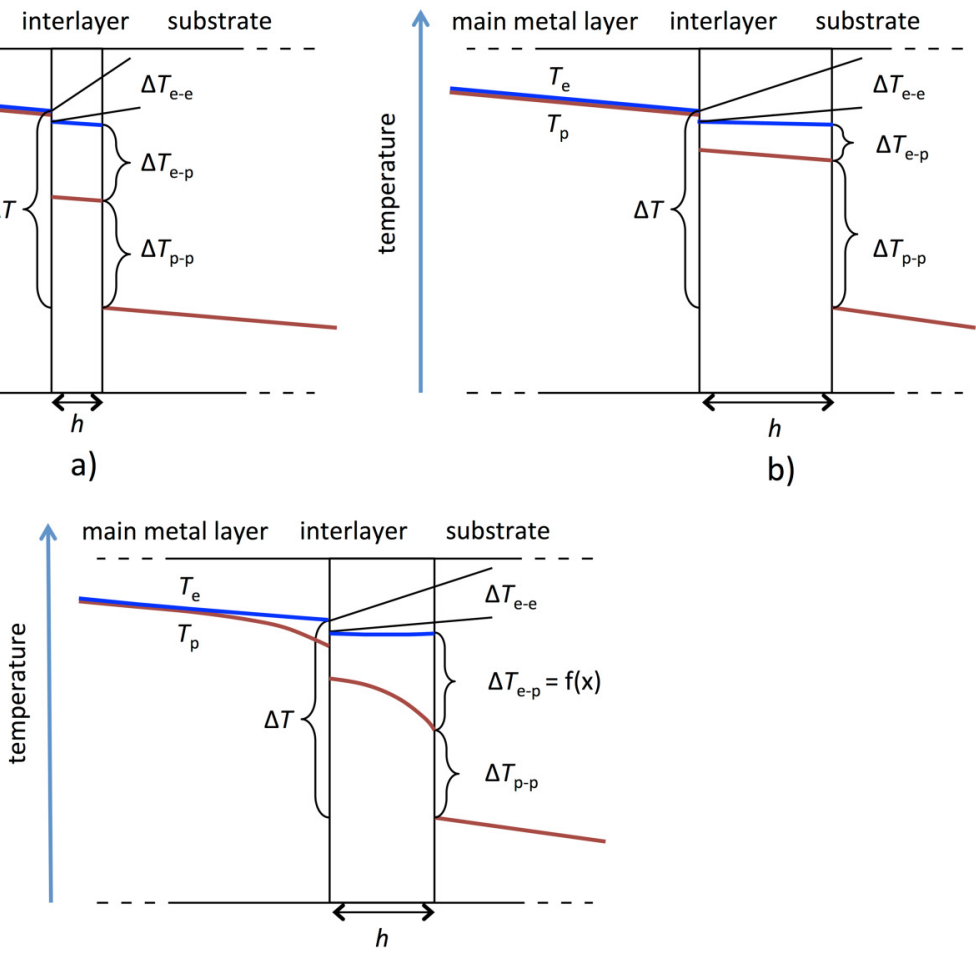

c)
FIG. 10. Schematic temperature profiles predicted by [(a) and (b)] the simplified steady-state model in Eq. (1) and (c) the rigorous 31-TTM model based on Eq. (22). Sketches in (a) and (b) indicate the difference between the phonon and electron temperatures for different interlayer thicknesses. 
interlayer, and a drop $\Delta T_{\mathrm{pp}}$ at the interface between the interlayer and the dielectric. For simplicity's sake, not much heed has been given to the evolution of the electron and phonon temperature in the interlayer. Rather, the difference in these two temperatures, $\Delta T_{\mathrm{ep}}$, has been considered constant corresponding to an average over the thickness of the layer. Under this assumption, the heat transferred from the electrons to the phonons is proportional to the thickness of the interlayer and to the temperature difference between electrons and phonons. Since the heat passing through the interface is proportional to the phonon-phonon TBC at the metal-dielectric interface and is identical to the heat transferred in the interlayer from the electrons to the phonons, the ratio $\Delta T_{\mathrm{ep}} / \Delta T_{\mathrm{pp}}$ becomes smaller and smaller with larger interlayer thickness and $\Delta T_{\mathrm{pp}}$ approaches asymptotically $\Delta T-\Delta T_{\text {ee }}$, cf. Fig. 10(b). In parallel, some phonons tunnel through the interlayer, yet this contribution gets negligible for interlayers thicker than $2 \mathrm{~nm}$.

As such this simple model has two major shortcomings: (i) it neglects the heat flux equilibrium and the ensuing evolution of the phonon and electron temperature in the interlayer and (ii) it does not give any description of the phonon-phonon heat transfer at the main layer/interlayer interface. In order to overcome these shortcomings, we detail in the following a 31-TTM which takes care of these issues.

\section{Two temperatures model for a 3-layers system}

In an attempt to provide a more rigorous approach to describe the mechanisms involved in the heat transfer of metal/(nanometer-thick) interlayer/dielectric system, we developed the formalism for a 31-TTM, schematically depicted in Fig. 10(c). Similarly to the TTM developed by Majumdar and Reddy, ${ }^{39}$ the 31 -TTM presented below assumes that

1. the heat transport through the metallic layers is due to the electron and phonon gases, as well as to their mutual interactions and

2. the system has reached a steady state.

After a few hundred picoseconds, the temperature distribution in a pump-probe experiment evolves much more slowly than the time constant of equilibration between electron and phonon temperature and hence might be approximated by a steady-state treatment.
A schematic of the system is presented in Fig. 11. The electron and phonon temperatures inside each layer obey the leading differential equations ${ }^{39,40}$

$$
\begin{gathered}
\frac{d^{2} T_{e i}}{d x^{2}}-\frac{G_{i}}{\kappa_{e i}}\left(T_{e i}-T_{p i}\right)=0, \\
\frac{d^{2} T_{p i}}{d x^{2}}+\frac{G_{i}}{\kappa_{p i}}\left(T_{e i}-T_{p i}\right)=0,
\end{gathered}
$$

with $T$ being the temperature, $G$ being the electron-phonon coupling factor, and $\kappa$ being the thermal conductivity. The subscripts $i$, $e$, and $p$ stand for the layer under consideration, the electrons, and the phonons, respectively. The general solutions of Eqs. (3) and (4) for the electron and phonon temperatures are given by

$$
\begin{aligned}
T_{e i}(x)= & A_{i}+B_{i}\left(x-l_{i-1}\right) \\
& +\frac{\kappa_{a i}}{\kappa_{e i}}\left(C_{i} \sinh \left(\frac{x-l_{i-1}}{d_{i}}\right)+D_{i} \cosh \left(\frac{x-l_{i-1}}{d_{i}}\right)\right), \\
T_{p i}(x)= & A_{i}+B_{i}\left(x-l_{i-1}\right) \\
& -\frac{\kappa_{a i}}{\kappa_{p i}}\left(C_{i} \sinh \left(\frac{x-l_{i-1}}{d_{i}}\right)+D_{i} \cosh \left(\frac{x-l_{i-1}}{d_{i}}\right)\right),
\end{aligned}
$$

where $A, B, C$, and $D$ are constants to be determined for each layer and

$$
\begin{gathered}
d_{i}^{2}=\frac{\kappa_{a i}}{G_{i}}, \\
\frac{1}{\kappa_{a i}}=\frac{1}{\kappa_{e i}}+\frac{1}{\kappa_{p i}} .
\end{gathered}
$$

Taking into account that the heat conduction in the dielectric layer is due to the phonon gas only, its temperature evolution is described by

$$
T_{3}(x)=A_{3}+B_{3} x
$$

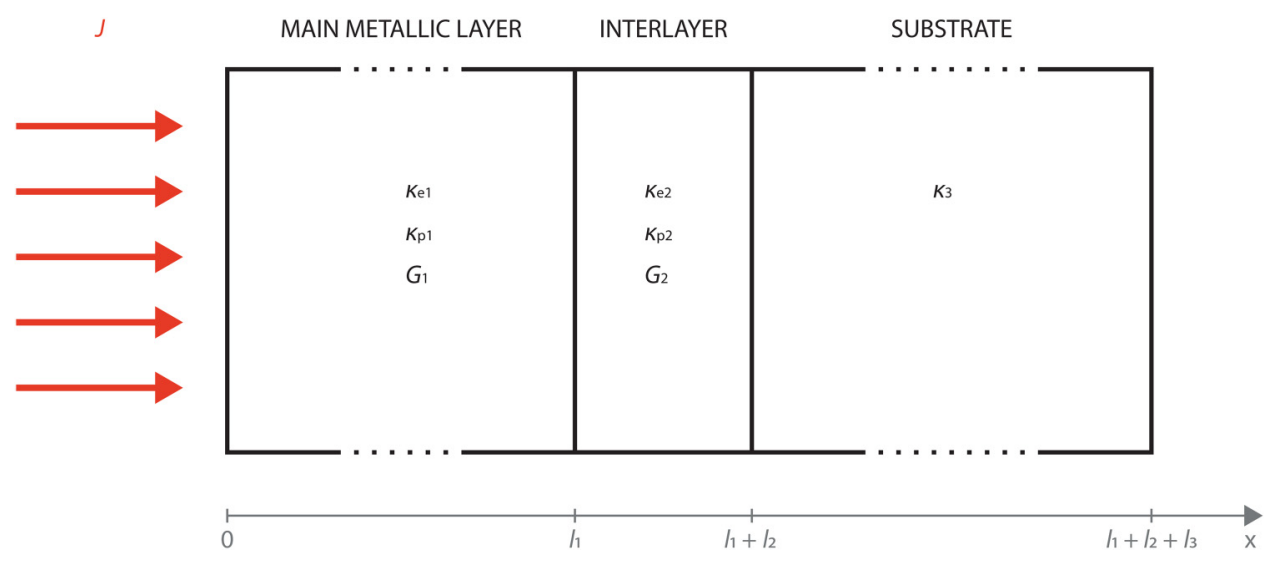

FIG. 11. System considered to develop the 3I-TTM. The heat flux first propagates through the two metallic layers before reaching the dielectric substrate of thermal conductivity $\kappa$. Each metallic layer is characterized by its electron $\left(\kappa_{\mathrm{e}}\right)$ and phonon $\left(\kappa_{\mathrm{p}}\right)$ thermal conductivities along with its electron-phonon coupling constant $\mathrm{G}$. 
The ten constants involved in Eqs. (5), (6), and (9) are determined by proper boundary conditions at the layer interfaces. Considering that the laser energy is only transferred to the electron gas and that the phonons at the illuminated film surface are insulated from the environment, the boundary conditions at $x=0$ are given by

$$
\begin{gathered}
\left.-\kappa_{e 1} \frac{d T_{e 1}}{d x}\right]_{x=0}=J \\
\left.\frac{d T_{p 1}}{d x}\right]_{x=0}=0 .
\end{gathered}
$$

At the interface $x=l_{1}$ between the main metallic layer and the interlayer, the electrons (phonons) of the main metallic layer transfer their energy to the electrons (phonons) of the interlayer. The electrons and the phonons are both assumed to encounter a certain interfacial resistance. Those conditions combined with the usual requirements for heat flux continuity lead to the following boundary conditions:

$$
\begin{gathered}
\left.\left.\kappa_{e 1} \frac{d T_{e 1}}{d x}\right]_{x=l_{1}}=\kappa_{e 2} \frac{d T_{e 2}}{d x}\right]_{x=l_{1}}, \\
\left.\left.\kappa_{p 1} \frac{d T_{p 1}}{d x}\right]_{x=l_{1}}=\kappa_{p 2} \frac{d T_{p 2}}{d x}\right]_{x=l_{1}}, \\
\left.\left.\left(T_{e 1}-T_{e 2}\right)\right]_{x=l_{1}}=-R_{\mathrm{e} 12} \kappa_{e 2} \frac{d T_{e 2}}{d x}\right]_{x=l_{1}}, \\
\left.\left.\left(T_{p 1}-T_{p 2}\right)\right]_{x=l_{1}}=-R_{\mathrm{p} 12} \kappa_{\mathrm{p} 2} \frac{d T_{\mathrm{p} 2}}{d x}\right]_{x=l_{1}},
\end{gathered}
$$

where $R_{\mathrm{e} 12}$ and $R_{\mathrm{p} 12}$ are the thermal resistances accounting for the electron-electron and phonon-phonon thermal mismatch at the interface of layers 1 and 2 .

At the interface $x=l_{1}+l_{2}$ between the interlayer and the substrate, the heat flux transported by electrons into the interlayer is assumed to have been transferred to the phonons of the interlayer, which then transfer their energy to the phonons of the substrate via phonon-phonon interactions. These conditions read as follows:

$$
\begin{gathered}
\left.\frac{d T_{e 2}}{d x}\right]_{x=l_{1}+l_{2}}=0, \\
\left.\left.\kappa_{p 2} \frac{d T_{p 2}}{d x}\right]_{x=l_{1}+l_{2}}=\kappa_{3} \frac{d T_{3}}{d x}\right]_{x=l_{1}+l_{2}}, \\
\left.\left.\left(T_{p 2}-T_{3}\right)\right]_{x=l_{1}+l_{2}}=-R_{23} \kappa_{3} \frac{d T_{3}}{d x}\right]_{x=l_{1}+l_{2}} .
\end{gathered}
$$

Finally, at the bottom at $x=l_{1}+l_{2}+l_{3}$ of the substrate, the temperature is assumed to remain constant and equal to room temperature, that is to say

$$
\left.T_{3}\right]_{x=l_{1}+l_{2}+l_{3}}=T_{0} .
$$

Equations (10)-(19) allow for calculating all the constants involved in Eqs. (5), (6), and (9). The $h_{\mathrm{BD}}=1 / R_{\mathrm{EFF}}$ of the three-layer system is then determined through its effective thermal resistance defined by

$$
T\left(l_{1}+l_{2}\right)-T_{3}\left(l_{1}+l_{2}\right)=R_{E F F} \cdot J
$$

where $T\left(l_{1}+l_{2}\right)$ is the common linear extrapolation of the electron and phonon temperatures of the interlayer and is therefore given by

$$
T\left(l_{1}+l_{2}\right)=A_{2}+B_{2} \cdot l_{2} .
$$

By inserting Eqs. (5), (6), and (9) into Eq. (21) and assuming that the first layer is much thicker than its electron-phonon coupling distance $\left(l_{1} / d_{1} \gg 1\right)$, as is the case for the layers of $\mathrm{Ag}\left(\mathrm{d}_{1}=14 \mathrm{~nm}\right)$ and $\mathrm{Au}\left(\mathrm{d}_{1}=10 \mathrm{~nm}\right)$ used in the work (see Table IV), one obtains

$$
R_{E F F}=R_{23}+\frac{\kappa_{\mathrm{a} 2}}{\kappa_{\mathrm{p} 2}}\left(\frac{a-b}{c}\right),
$$

where

$$
\begin{gathered}
a=\left(\frac{d_{1}}{\kappa_{a 1}} \frac{\kappa_{\mathrm{e} 2}}{\kappa_{2}}+\frac{\kappa_{\mathrm{a} 2}}{\kappa_{\mathrm{p} 2}}\left(R_{e 12}+R_{p 12}\right)\right) \cosh \left(\frac{l_{2}}{d_{2}}\right) \\
+\frac{d_{2}}{\kappa_{\mathrm{p} 2}} \sinh \left(\frac{l_{2}}{d_{2}}\right), \\
b=\frac{1}{\kappa_{2}}\left(R_{\mathrm{e} 12} \kappa_{\mathrm{e} 2}-R_{\mathrm{p} 12} \kappa_{\mathrm{p} 2}+\alpha_{21} d_{1}\right), \\
c=\frac{\kappa_{\mathrm{a} 2}}{d_{2}}\left(\frac{d_{1}}{\kappa_{a 1}}+R_{\mathrm{e} 12}+R_{\mathrm{p} 12}\right) \sinh \left(\frac{l_{2}}{d_{2}}\right)+\cosh \left(\frac{l_{2}}{d_{2}}\right), \\
\alpha_{21}=\frac{\kappa_{\mathrm{e}_{1}}}{\kappa_{\mathrm{e}_{2}}}-\frac{\kappa_{\mathrm{p}_{1}}}{\kappa_{\mathrm{p}_{2}}}
\end{gathered}
$$

TABLE IV. Electronic thermal conductivity, lattice thermal conductivity, and electron-phonon coupling factor at $T_{\mathrm{e}}=300 \mathrm{~K}$ for all the metals tested.

\begin{tabular}{lccccccccc}
\hline \hline & $\mathrm{Ag}$ & $\mathrm{Au}$ & $\mathrm{Cu}$ & $\mathrm{Mo}$ & $\mathrm{Ni}$ & $\mathrm{Si}$ & $\mathrm{Al}_{2} \mathrm{O}_{3}$ & $\mathrm{C}$ (diamond) \\
\hline$\kappa_{\mathrm{e}}(\mathrm{W} / \mathrm{m} \mathrm{K})$ & 424 & 314 & 384 & 105 & 66 & $\ldots$ & $\ldots$ & $\ldots$ \\
$\kappa_{\mathrm{p}}(\mathrm{W} / \mathrm{m} \mathrm{K})$ & $5.2^{\mathrm{a}}$ & $2.6^{\mathrm{a}}$ & $17^{\mathrm{a}}$ & $33^{\mathrm{b}}$ & $25^{\mathrm{a}}$ & 124 & 35 & 1500 \\
$\mathrm{G} 10^{16}\left(\mathrm{~W} / \mathrm{m}^{3} \mathrm{~K}\right)$ & 2.5 & 2.6 & $10^{\mathrm{c}}$ & 48 & 100 & $\ldots$ & $\ldots$ & $\ldots$ \\
\hline \hline
\end{tabular}

${ }^{\mathrm{a}}$ From Ref. 36

${ }^{\mathrm{b}}$ From Ref. 37 .

${ }^{\mathrm{c}}$ From Ref. 30 . 
Given that the microscopic electron-phonon interactions require a space to take place, in general, the TTM based on Fourier's equation is typically considered suitable to describe the heat conduction in metallic layers with thicknesses greater than the mean free path of their electrons and phonons. This constraint was the assumption done by Qiu and Tien ${ }^{41}$ to derive this model from the Boltzmann transport equation, by evaluating its scattering term using quantum mechanics and statistical considerations. If one, however, considers the equivalent temperature, i.e., the mean temperature of heat carriers being present at a point in space that do not obey an equilibrium distribution in heat as has been proposed by Chen and Zeng $^{42,43}$ to be used in the context of the Boltzmann Transport Equation, the guiding differential equations for that equivalent temperature will be very similar to those obtained from Fourier's law. Furthermore, boundary resistances manifest by temperature jumps at the interface obtain naturally from this analysis as well. While the parameters of the solution of the set of differential equations may differ slightly in their analytical value, the outcome for the equivalent temperature evolution will be very similar to the one obtained based on the analysis presented here.

The parameters required to compare the prediction of the model in Eq. (22) to the experimental data are $\kappa_{\mathrm{e}}, \kappa_{\mathrm{p}}$, and $G$ of each layer, as well as $R_{\mathrm{e} 12}, R_{\mathrm{p} 12}$, and $R_{23}$. The values of $\kappa_{\mathrm{e}}, \kappa_{\mathrm{p}}$, and $G$ are tabulated in Table IV, while those of $R_{\mathrm{e} 12}$ and $R_{\mathrm{p} 12}$ are summarized in Table V. All $R_{23}$ values were taken from Ref. $25, \kappa_{\mathrm{e}}$ was calculated by subtracting the lattice thermal conductivity $\kappa_{\mathrm{p}}$ from the thermal conductivity reported in Table I and $G$ was taken from the work reported by Lin et al., ${ }^{29}$ assuming $T_{\mathrm{e}} \approx 300 \mathrm{~K}$. $R_{\mathrm{e} 12}$ was estimated by using the procedure described in Sec. IV A, while $R_{\mathrm{p} 12}$ was calculated through the standard DMM involving a Debye linear dispersion relation.

The fitting of Eq. (22) to the experimental data was performed by allowing the TBC values to vary within $20 \%$ of the ones given in Ref. 25, while the electron-phonon coupling factor was allowed to vary by a factor of 2 as compared to the value given in Table IV. Figures 3-5 and 7-9 display the fits of Eqs. (1) and (22) along with the experimental data. It is worthwhile noting that the present analysis is coherent with the results of all systems investigated without recurring to a fitting parameter. For systems with a $\mathrm{Cu}$ interlayer, the fits obtained by assuming the interlayer to have the same electron-phonon coupling factor as $\mathrm{Mo}$ and $\mathrm{Ni}$ are further provided for comparison. Similarly, for systems with Mo or Ni interlayers, fits that assume the interlayer to have the same electron-phonon coupling factor as copper are provided for comparison. An accurate fit is thus obtained for all cases, with the predictions of both Eqs. (1) and (22) falling close to

TABLE V. $1 / R_{\mathrm{e} 12}$ values calculated from the electronic equivalent of the DMM and $1 / R_{\mathrm{p} 12}$ values calculated from the DMM for the different metal-metal couples tested at $300 \mathrm{~K}$.

\begin{tabular}{lcc}
\hline \hline Metal couple & $\begin{array}{c}1 / R_{\mathrm{e} 12} \\
\left(\mathrm{GW} / \mathrm{m}^{2} \mathrm{~K}\right)\end{array}$ & $\begin{array}{c}1 / R_{\mathrm{p} 12} \\
\left(\mathrm{GW} / \mathrm{m}^{2} \mathrm{~K}\right)\end{array}$ \\
\hline $\mathrm{Au}-\mathrm{Cu}$ & 4.5 & 0.24 \\
$\mathrm{Ag}-\mathrm{Ni}$ & 4.6 & 0.25 \\
$\mathrm{Au}-\mathrm{Ni}$ & 5.1 & 0.15 \\
$\mathrm{Ag}-\mathrm{Mo}$ & 4.5 & 0.22 \\
\hline \hline
\end{tabular}

each other. The fits obtained using larger (for $\mathrm{Cu}$ interlayers) and smaller (for Mo and Ni interlayers) electron-phonon coupling factors further show features that are completely different from the experimental data, which confirms that the overall TBC is controlled by the interlayer electron-phonon coupling factor.

Figure 12 details this last statement by means of a temperature profile obtained for both a thin and a large interlayer that has either a small $\left(5.5 \mathrm{MW} / \mathrm{m}^{3} \mathrm{~K}\right)$ or a large $\left(55 \mathrm{MW} / \mathrm{m}^{3} \mathrm{~K}\right)$ electron-phonon coupling factor. As expected, nonequilibrium between electrons and phonons is larger in thin interlayers, resulting in lower TBC values. A large electron-phonon coupling factor is shown to be an efficient way to reduce nonequilibrium even in very thin interlayers, which is consistent with the observation that the interlayer thickness required for the TBC to reach a plateau decreases with increasing electron-phonon coupling factors.

\section{E. Low-temperature data on Au/(nanometer-thick) $\mathrm{Cu} / \mathrm{Si}$ interfaces}

As expected at such a temperature, the TBC values are lower by a factor of roughly 2 as compared to room temperature values (see Fig. 9). Interestingly enough, however, the overall dependence of the TBC on the interlayer thickness is observed to be very similar at $80 \mathrm{~K}$ and at room temperature, i.e., the TBC increases monotonically over roughly $10 \mathrm{~nm}$ before reaching a plateau.

A quantitative analysis of these data was achieved using the fitting models described by Eqs. (1) and (22). At $80 \mathrm{~K}$, the parameters necessary to perform the fits were determined as follows:

- The thermal resistance due to the electron temperature mismatch at the $\mathrm{Au} / \mathrm{Cu}$ interface $R_{\mathrm{e} 12}$ [for Eq. (22)] $=1 / h_{\mathrm{ee}}$ [in Eq. (1)] was evaluated to be $1.02 \mathrm{GW} / \mathrm{m}^{2} \mathrm{~K}$ using Eq. (2).

- The thermal resistance due to the phonon temperature mismatch at the $\mathrm{Au} / \mathrm{Cu}$ interface $R_{\mathrm{p} 12}$ was evaluated to be $0.15 \mathrm{GW} / \mathrm{m}^{2} \mathrm{~K}$ using a standard DMM with a linear Debye dispersion relationship with the sound velocities extracted from the elastic constants measured at $80 \mathrm{~K}^{44,45}$

- The phonon thermal conductivity is expected to be inversely proportional to the absolute temperature and was thus calculated from room temperature values and set to 9.8 and $63 \mathrm{~W} / \mathrm{mK}$ for gold and copper, respectively.

- The electron thermal conductivity was calculated by subtracting the phonon thermal conductivity from the thermal conductivity given in Table I.

According to basic theory ${ }^{46}$ on electron-phonon heat transfer, the coupling factor $G$ should be independent of temperature for phonon and electron temperatures lower, or on the order of, the Debye-temperature of the lattice. The 31-TTM and the simpler model given by Eq. (1) would hence suggest that the interlayer thickness $l_{\text {trans }}$ at which the TBC should reach a stable value should scale roughly as

$$
l_{\text {trans }} \propto \frac{h_{\mathrm{pp}}}{G} .
$$

Since $h_{\mathrm{pp}}$ decreases by a factor of about 2 between $300 \mathrm{~K}$ and $80 \mathrm{~K}$, the basic model given by Eq. (1) would predict the transition to 

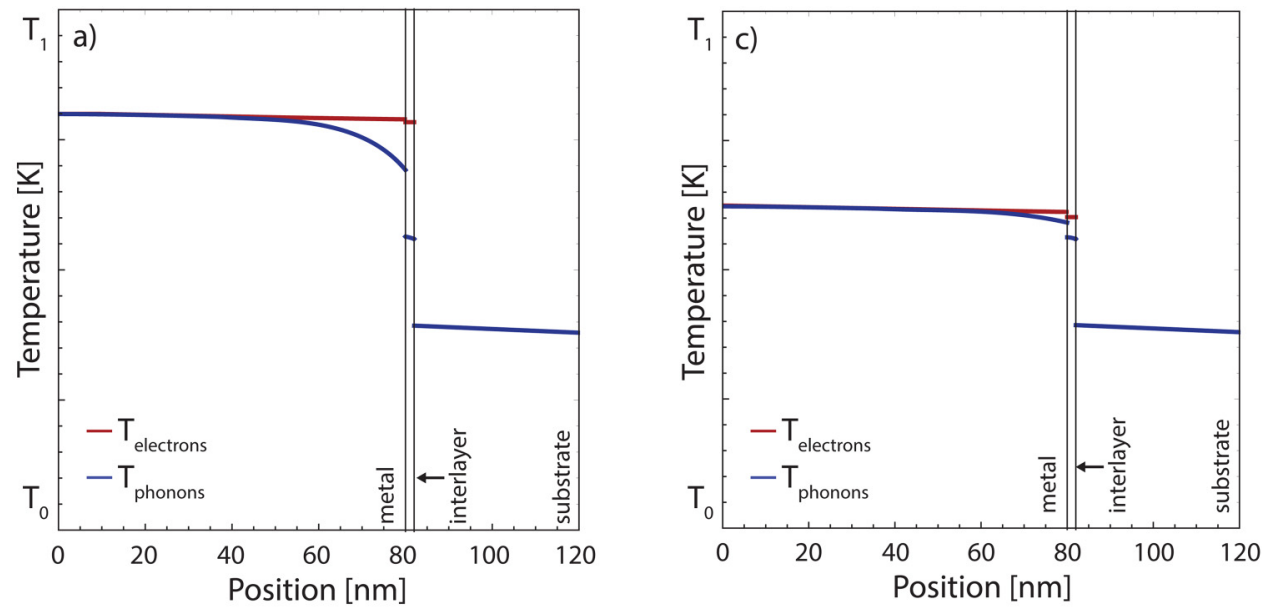

FIG. 12. Temperature profiles predicted by the 31-TTM for a system with an interlayer characterized by $(d$, $\mathrm{G})=(\mathrm{a})\left(2 \mathrm{~nm}, 5 \times 10^{16} \mathrm{MW} / \mathrm{m}^{3} \mathrm{~K}\right)$, (b) $\left(20 \mathrm{~nm}, 5 \times 10^{16} \mathrm{MW} / \mathrm{m}^{3} \mathrm{~K}\right)$, (c) $(2 \mathrm{~nm}$, $\left.50 \times 10^{16} \mathrm{MW} / \mathrm{m}^{3} \mathrm{~K}\right)$, and (d) $(20 \mathrm{~nm}$, $\left.50 \times 10^{16} \mathrm{MW} / \mathrm{m}^{3} \mathrm{~K}\right)$. All calculations were done with the same values of $T_{0}$ and $T_{1}$ driving the heat flux $\mathrm{J}$.
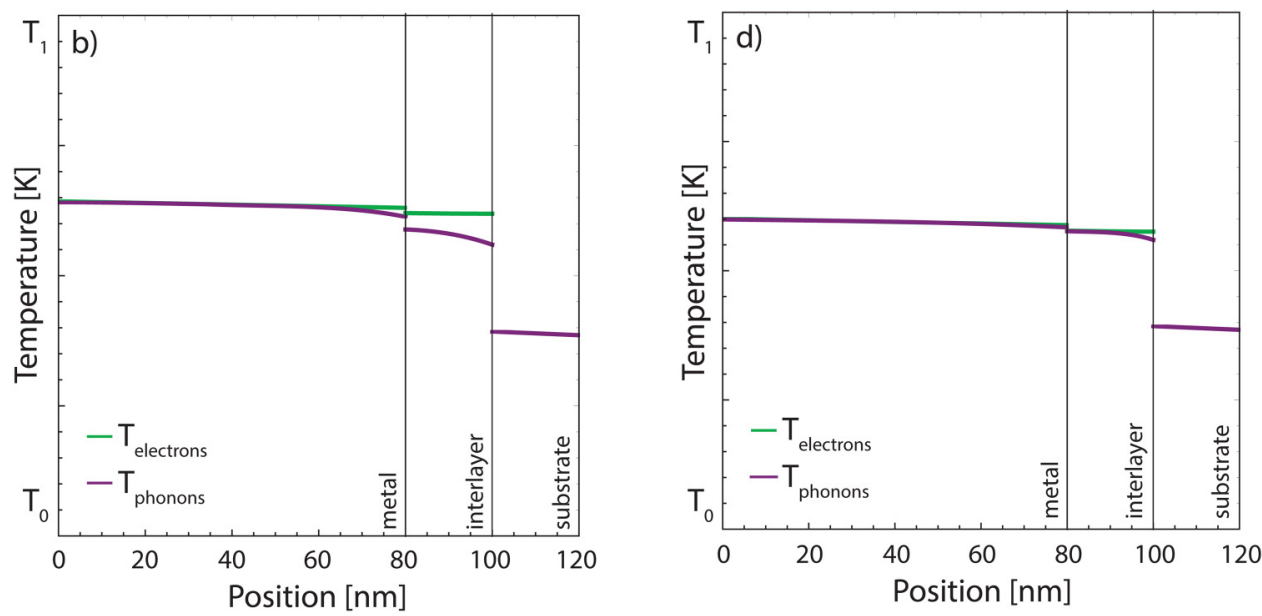

happen over a thickness range smaller by the same factor. The 31-TTM can also account for changes in phonon conductivity, in $h_{\mathrm{ee}}$, and in temperature distribution, making it more complete. For the room temperature value of $G$, the 3l-TTM also predicts a steeper rise in TBC with increasing interlayer thickness at $80 \mathrm{~K}$ than for $300 \mathrm{~K}$. This, however, would be qualitatively at odds with the observed changes for the $\mathrm{Au} /$ (nanometer-thick) $\mathrm{Cu} / \mathrm{Si}$ system studied at both $300 \mathrm{~K}$ and $80 \mathrm{~K}$, cf. Figs. 6 and 9, where, experimentally, the transition seems to take place over even a slightly larger thickness range at $80 \mathrm{~K}$ than at $300 \mathrm{~K}$. The predictions of the 31-TTM with an identical electron-phonon coupling factor for both temperatures are also indicated. In order to make the 31-TTM model fit, the experimental evolution one would have to reduce the electron-phonon coupling constant by a factor of roughly 2. This stands to reason since comparable changes in electron-phonon coupling factors have experimentally been observed between $300 \mathrm{~K}$ and $100 \mathrm{~K}$ for gold and silver by Groeneveld et al. ${ }^{47}$ and are in agreement with time dependent density functional theory modeling for gold by Zhou et al. ${ }^{48}$ In the absence of comparable data for $\mathrm{Cu}$, yet in view of the similarity of the electron structure of $\mathrm{Ag}, \mathrm{Au}$, and $\mathrm{Cu}$, we infer that copper would experience a similar reduction in the electron-phonon coupling factor.

Since the electron-phonon coupling factor had already been determined by the fit of the data measured at $300 \mathrm{~K}$, fitting of the experimental data was performed by setting the electron-phonon coupling constant to half its room temperature value and allowed the intrinsic TBC at the interface between metal interlayer and dielectric to vary by $20 \%$. The results obtained are plotted with the experimental results in Fig. 6, showing good agreement.

\section{F. On the utility of nanometer-thick interlayers in real applications}

In this section, we discuss the influence of an interlayer on the thermal transport of a metal/dielectric system in its globality. This work shows that a thin interlayer can significantly enhance the $\mathrm{TBC}$ at the interface between the metallic stack and the dielectric. These results were obtained by assuming that the system was made 
of three metallic layers and one substrate. The interface was thus defined as being the plane that separates the interlayer from the substrate (configuration I in Fig. 13). The interlayer is, however, known to be responsible for an additional thermal resistance that scales with its thickness and the inverse of its thermal conductivity, and it is thus likely to reduce the overall thermal transport efficiency of the system. To account for these two opposite trends (i.e., an improved TBC vs an additional diffusive thermal resistance within the interlayer), the overall thermal boundary conductance $\mathrm{TBC}_{\text {global }}$ of the $\mathrm{Au} /($ nanometer-thick $\mathrm{Cu}) / \mathrm{Si}$ system was extracted from TDTR measurements, by assuming that the system is made of two metallic layers and one substrate. The interface was thus defined as being the interlayer together with the plane that separates the interlayer from the substrate (configuration II in Fig. 13).
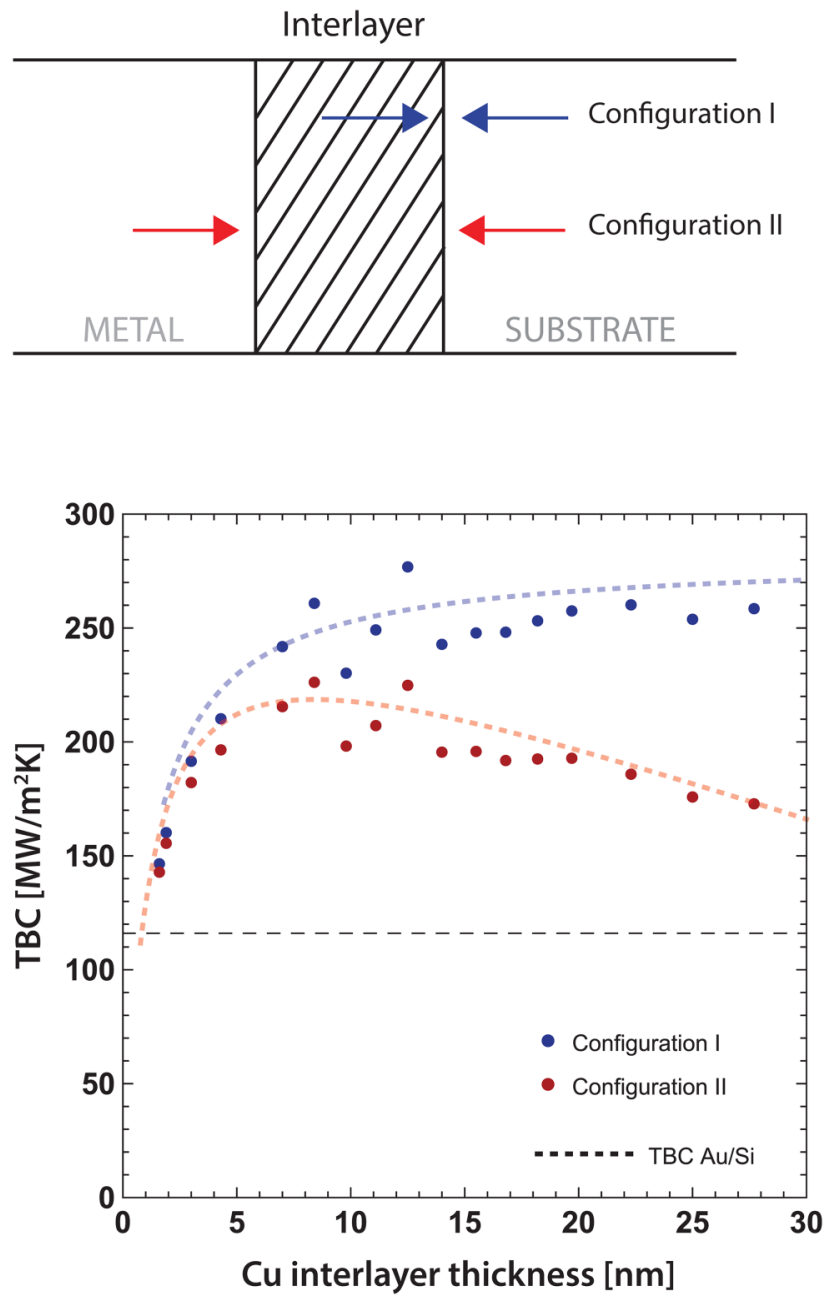

FIG. 13. Evolution of the TBC of an Au/Cu/Si sample as a function of the thickness of its $\mathrm{Cu}$ interlayer. The dots stand for the representative experimental data, while the blue and red dashed lines are eye guides related to the configurations I and II, respectively.
$\mathrm{TBC}_{\text {global }}$ was observed to increase before reaching a maximum at $220 \mathrm{MW} / \mathrm{m}^{2} \mathrm{~K}$ that is located at the beginning of the TBC plateau. In this region, the increase in the TBC dominates over the temperature drop within the interlayer, which is very small. For thicker layers, the TBC becomes constant, while the temperature drop within the interlayer increases, which results in a decrease of $\mathrm{TBC}_{\text {global }}$. The most efficient design for heat transport is thus achieved for interlayers that have a thickness that corresponds to the beginning of the plateau. The measurements performed, however, highlight that the range of thicknesses over which the interlayer is beneficial is relatively large, since $\mathrm{TBC}_{\text {global }}$ is larger than the TBC at the $\mathrm{Au} / \mathrm{Si}$ interface for all the thicknesses tested.

\section{CONCLUSION}

$\mathrm{Ni}$ and Mo nanometric interlayers have been shown to significantly increase the $\mathrm{TBC}$ of $\mathrm{Ag} / \mathrm{O}: \mathrm{C}$ and $\mathrm{Au} / \mathrm{O}: \mathrm{C}$ interfaces, through values that do not evolve much with the interlayer thickness. The TBC of both systems reaches a plateau at a value much higher than their corresponding TBC without interlayer, but somewhat below the ones of $\mathrm{Ni} / \mathrm{O}: \mathrm{C}$ and Mo/O:C. By contrast, the TBC of $\mathrm{Au} /($ nanometer-thick $\mathrm{Cu}$ )/dielectric systems has been shown to monotonically increase with the interlayer thickness over about $10 \mathrm{~nm}$ before reaching a plateau due to the relatively weaker electron-phonon coupling factor of $\mathrm{Cu}$ with respect to that of Mo and Ni. Furthermore, the comparison of the predictions of a threelayer two-temperature model with data measured at low temperature suggests that the electron-phonon coupling factor of $\mathrm{Cu}$ is reduced by roughly a factor of two, when its temperature drops down from $300 \mathrm{~K}$ to $80 \mathrm{~K}$.

\section{SUPPLEMENTARY MATERIAL}

See the supplementary material for a detailed analysis of TDTR measurement sensitivity to the input and fitting parameters used in the thermal model.

\section{ACKNOWLEDGMENTS}

The authors are grateful to the SNSF (Project No. 200021_149290) for its financial support and to Professor O. Martin from the Nanophotonics and Metrology Laboratory (NAM, EPFL) for providing the laser source. Dr. A. Magrez and Dr. C. Monachon from the iPhys platform (EPFL) are warmly thanked for their support with the Empyrean diffractometer and for having provided the code to analyze the TDTR data, respectively.

\section{REFERENCES}

${ }^{1}$ H. K. Lyeo and D. G. Cahill, "Thermal conductance of interfaces between highly dissimilar materials," Phys. Rev. B 73(14), 144301 (2006).

${ }^{2} \mathrm{G}$. Chen, "Heat transfer in micro- and nanoscale photonic devices," Annu. Rev. Heat Transfer 7(7), 1-57 (1996).

${ }^{3}$ C. Monachon, L. Weber, and C. Dames, "Thermal boundary conductance: A materials science perspective," Annu. Rev. Mater. Res. 46, 433-463 (2016).

${ }^{4}$ C. Monachon and L. Weber, "Influence of diamond surface termination on thermal boundary conductance between $\mathrm{Al}$ and diamond," J. Appl. Phys. 113(18), 183504 (2013). 
${ }^{5}$ D. W. Oh, S. Kim, J. A. Rogers, D. G. Cahill, and S. Sinha, "Interfacial thermal conductance of transfer-printed metal films," Adv. Mater. 23(43), 5028-5033 (2011).

${ }^{6}$ K. C. Collins, S. Chen, and G. Chen, "Effects of surface chemistry on thermal conductance at aluminum-diamond interfaces," Appl. Phys. Lett. 97(8), 083102 (2010).

${ }^{7}$ P. E. Hopkins, L. M. Phinney, J. R. Serrano, T. E. Beechem, "Effects of surface roughness and oxide layer on the thermal boundary conductance at aluminum/ silicon interfaces," in 2010 14th International Heat Transfer Conference (American Society of Mechanical Engineers, 2010).

${ }^{8}$ J. C. Duda and P. E. Hopkins, "Systematically controlling Kapitza conductance via chemical etching," Appl. Phys. Lett. 100(11), 111602 (2012).

${ }^{9}$ W. Ih Choi, K. Kim, and S. Narumanchi, "Thermal conductance at atomically clean and disordered silicon/aluminum interfaces: A molecular dynamics simulation study," J. Appl. Phys. 112(5), 054305 (2012).

${ }^{10}$ A. J. Schmidt, K. C. Collins, A. J. Minnich, and G. Chen, "Thermal conductance and phonon transmissivity of metal-graphite interfaces," J. Appl. Phys. 107(10), 104907 (2010).

${ }^{11}$ J. C. Duda, C. Y. Yang, B. M. Foley, R. Cheaito, D. L. Medlin, R. E. Jones, and P. E. Hopkins, "Influence of interfacial properties on thermal transport at gold: Silicon contacts," Appl. Phys. Lett. 102(8), 081902 (2013).

${ }^{12} \mathrm{Y}$. Wang, Z. Lu, A. K. Roy, and X. Ruan, "Effect of interlayer on interfacial thermal transport and hot electron cooling in metal-dielectric systems: An electron-phonon coupling perspective," J. Appl. Phys. 119(6), 065103 (2016). ${ }^{13}$ M. Jeong, J. P. Freedman, H. J. Liang, C. M. Chow, V. M. Sokalski, J. A. Bain, and J. A. Malen, "Enhancement of thermal conductance at metal-dielectric interfaces using subnanometer metal adhesion layers," Phys. Rev. Appl. 5(1), 014009 (2016).

${ }^{14} \mathrm{M}$. Blank and L. Weber, "Influence of the thickness of a nanometric copper interlayer on Au/dielectric thermal boundary conductance," J. Appl. Phys. 124(10), 105304 (2018)

${ }^{15} \mathrm{M}$. Björck and G. Andersson, "Genx: An extensible X-ray reflectivity refinement program utilizing differential evolution," J. Appl. Crystallogr. 40(6), 1174-1178 (2007).

${ }^{16}$ K. Kang, Y. K. Koh, C. Chiritescu, X. Zheng, and D. G. Cahill, "Two-tint pump-probe measurements using a femtosecond laser oscillator and sharp-edged optical filters," Rev. Sci. Instrum. 79(11), 114901 (2008).

${ }^{17} \mathrm{C}$. Monachon, "Thermal boundary conductance between metals and dielectrics," Ph.D. Thesis No. 5872 (EPFL, 2013).

${ }^{18}$ D. G. Cahill, "Analysis of heat flow in layered structures for time-domain thermoreflectance," Rev. Sci. Instrum. 75(12), 5119-5122 (2004).

19. Rumble, CRC Handbook of Chemistry and Physics (CRC Press, 2017), ISBN:978-1498784542.

${ }^{20}$ R. L. Powell and W. A. Blanpied, Thermal Conductivity of Metals and Alloys at low Temperatures: A Review of the Literature (No. NBS-CIRC-556) (National Bureau of Standards, Gaithersburg, MD, 1954).

${ }^{21} \mathrm{C}$. T. Anderson, "The heat capacities of chromium, chromic oxide, chromous chloride and chromic chloride at low temperatures," J. Am. Chem. Soc. 59(3), 488-491 (1937).

${ }^{22}$ Y. Takahashi and H. Akiyama, "Heat capacity of gold from 80 to $1000 \mathrm{~K}$," Thermochim. Acta 109(1), 105-109 (1986).

${ }^{23} \mathrm{G}$. K. White and S. J. Collocott, "Heat capacity of reference materials: $\mathrm{Cu}$ and W," J. Phys. Chem. Ref. Data 13(4), 1251-1257 (1984).

${ }^{24}$ Y. Yamamoto, T. Imai, K. Tanabe, T. Tsuno, Y. Kumazawa, and N. Fujimori, "The measurement of thermal properties of diamond," Diam. Relat. Mater. 6(8), 1057-1061 (1997)

${ }^{25} \mathrm{M}$. Blank and L. Weber, "Towards a coherent database of thermal boundary conductance at metal/dielectric interfaces," J. Appl. Phys. 125(9), 095302 (2019).
${ }^{26}$ R. B. Wilson and D. G. Cahill, "Anisotropic failure of Fourier theory in timedomain thermoreflectance experiments," Nat. Commun. 5, 5075 (2014).

${ }^{27} \mathrm{~S}$. Li, Q. Zheng, Y. Lv, X. Liu, X. Wang, P. Y. Huang, G. Cahill, and B. Lv, "High thermal conductivity in cubic boron arsenide crystals," Science 361(6402), 579-581 (2018).

${ }^{28}$ R. B. Wilson and D. G. Cahill, "Limits to Fourier theory in high thermal conductivity single crystals," Appl. Phys. Lett. 107(20), 203112 (2015).

${ }^{29} \mathrm{Z}$. Lin, L. V. Zhigilei, and V. Celli, "Electron-phonon coupling and electron heat capacity of metals under conditions of strong electron-phonon nonequilibrium," Phys. Rev. B 77(7), 075133 (2008).

${ }^{30}$ H. E. Elsayed-Ali, T. B. Norris, M. A. Pessot, and G. A. Mourou, "Time-resolved observation of electron-phonon relaxation in copper," Phys. Rev. Lett. 58(12), 1212 (1987).

${ }^{31}$ P. H. Dederichs, H. Schober, and D. J. Sellmyer, Metals: Phonon States, Electron States and Fermi Surfaces (Springer-Verlag, 1981), ISBN:978-3-540-09774-7.

${ }^{32}$ W. Martienssen and H. Warlimont, Springer Handbook of Condensed Matter and Materials Data (Springer Science \& Business Media, 2006), ISBN:3-540-44376-2.

${ }^{33}$ B. C. Gundrum, D. G. Cahill, and R. S. Averback, "Thermal conductance of metal-metal interfaces,” Phys. Rev. B 72(24), 245426 (2005).

${ }^{34}$ C. Kittel, Introduction to Solid State Physics (John Wiley \& Sons. Inc., New York, 2005), ISBN: 0-471-41526-X.

${ }^{35}$ D. Gall, "Electron mean free path in elemental metals," J. Appl. Phys. 119(8), 085101 (2016)

${ }^{36}$ J. Hohlfeld, S. S. Wellershoff, J. Güdde, U. Conrad, V. Jähnke, and E. Matthias, "Electron and lattice dynamics following optical excitation of metals," Chem. Phys. 251(1-3), 237-258 (2000).

${ }^{37}$ S. S. Wellershoff, J. Gudde, J. Hohlfeld, J. G. Muller, E. Matthias, "Role of electron-phonon coupling in femtosecond laser damage of metals," in High-Power Laser Ablation (International Society for Optics and Photonics, 1998), Vol. 3343, pp. 378-388.

${ }^{38}$ E. Beaurepaire, J. C. Merle, A. Daunois, and J. Y. Bigot, "Ultrafast spin dynamics in ferromagnetic nickel," Phys. Rev. Lett. 76(22), 4250 (1996).

${ }^{39} \mathrm{~A}$. Majumdar and P. Reddy, "Role of electron-phonon coupling in thermal conductance of metal-nonmetal interfaces," Appl. Phys. Lett. 84(23), 4768-4770 (2004). ${ }^{40} \mathrm{~J}$. Ordonez-Miranda, J. J. Alvarado-Gil, and R. Yang, "The effect of the electron-phonon coupling on the effective thermal conductivity of metal-nonmetal multilayers," J. Appl. Phys. 109(9), 094310 (2011).

${ }^{41}$ T. Q. Qiu and C. L. Tien, "Heat transfer mechanisms during short-pulse laser heating of metals," J. Heat Transfer 115(4), 835-841 (1993).

${ }^{42} \mathrm{G}$. Chen and T. Zeng, "Nonequilibrium phonon and electron transport in heterostructures and superlattices," Microscale Thermophys. Eng. 5(2), 71-88 (2001).

${ }^{43} \mathrm{~T}$. Zeng and G. Chen, "Phonon heat conduction in thin films: Impacts of thermal boundary resistance and internal heat generation," J. Heat Transfer 123(2), 340-347 (2001).

${ }^{44} \mathrm{~J}$. R. Neighbours and G. A. Alers, "Elastic constants of silver and gold," Phys. Rev. 111(3), 707 (1958)

${ }^{45} \mathrm{~W}$. C. Overton Jr, and J. Gaffney, "Temperature variation of the elastic constants of cubic elements. I. Copper," Phys. Rev. 98(4), 969 (1955).

${ }^{46}$ M. I. Kaganov, E. M. Lifshitz, and L. V. Tanatarov, "Relaxation between electrons and the crystalline lattice," J. Exp. Theor. Phys. 4, 173-178 (1957).

${ }^{47}$ R. H. Groeneveld, R. Sprik, and A. Lagendijk, "Femtosecond spectroscopy of electron-electron and electron-phonon energy relaxation in $\mathrm{Ag}$ and $\mathrm{Au}$," Phys. Rev. B 51(17), 11433 (1995).

${ }^{48}$ X. Zhou, L. Li, H. Dong, A. Giri, P. E. Hopkins, and O. V. Prezhdo, "Temperature dependence of electron-phonon interactions in gold films rationalized by time-domain ab initio analysis," J. Phys. Chem. C 121(32) 17488-17497 (2017). 